\title{
A009
}

\section{Segmenting Plays - Advantages of a Fully} Quantitative Approach

P.J. Brown* (Rose and Associates), R. Young (Rose and Associates), G. Citron (Rose and Associates) \& I. Longley (GIS-pax, Pty. Ltd)

\section{SUMMARY}

As industry has returned to basic exploration principles, play mapping is again a standard part of the exploration process. Many different methods are now employed for estimating and conveying the chance of geologic success for plays, or play segments, in map form. 


\begin{abstract}
As industry has returned to basic exploration principles, play mapping is again a standard part of the exploration process. Many different methods are now employed for estimating and conveying the chance of geologic success for plays, or play segments, in map form.
\end{abstract}

The most common approaches are:

1. Creating "traffic light" mapping methods expressing relative probability.

2. Higher fidelity versions of the method above, using shades of red/ yellow/green, reflecting different combinations of component maps.

3. Rules-driven programs that calculate average prospect Pg (chance of geologic success) for each play segment.

4. Mapping methods that apportion chance into play-specific and prospect- specific (conditional) probability, which are then convolved into maps of average prospect Pg (often called Total Chance in this approach).

Different exploration (and software) companies have landed upon each approach. The authors strongly advocate using method 4, since there is substantial value added to exploration decision-making with little incremental work. Using a series of map examples, this presentation will demonstrate the key advantages of a fully quantitative approach, including:

1. Providing requisite inputs for calculating both success case and probability-weighted Yet-to-Find volumes.

2. Providing requisite inputs for calculating the chance that a company can successfully 'open' a given play (through a discovery), based upon its corporate risk tolerance.

3. Guiding selection of the most appropriate prospects to drill with the initial test program, which differs significantly from proven to unproven plays/segments.

4. Understanding the potential uplift in chance of success for the remaining family of undrilled prospects in the play, or play segment from the next (or first) successful discovery.

5. Demonstrating the impact of item 4 upon portfolio aggregation of undrilled prospect volumes and chance. If shared/play and prospect-specific chance factors are not subdivided, the chance of at least one discovery (portfolio success) will be overstated, and every volume value (e.g., P90/50/10) that is calculated will wrong (P90's too low, P10's too high), except for the chance-weighted mean volume. 